\title{
Screening of differentially methylated genes in breast cancer and risk model construction based on TCGA database
}

\author{
LIANG FENG $^{*}$ and FENG JIN* \\ Department of Breast Surgery, The First Affiliated Hospital of China Medical University, \\ Shenyang, Liaoning 110001, P.R. China
}

Received June 13, 2018; Accepted September 10, 2018

DOI: $10.3892 / \mathrm{ol} .2018 .9457$

\begin{abstract}
Differentially methylated genes in breast cancer were screened out and a prognostic risk model of breast cancer was constructed. RNA-seq data and methylation data for breast cancer-related level 3 were downloaded from The Cancer Genome Atlas (TCGA), and MethylMix R package was used to screen out differentially methylated genes in cancer tissues and normal tissues. DAVID was used to analyze the GO enrichment of differentially methylated genes, ConsensusPathDB to analyze the PATHWAY pathways of differentially methylated genes, the single factor, multivariate Cox analysis and Akaike Information Criterion (AIC) to construct the prognostic risk model of breast cancer, and the ROC curve to judge the clinical application value of the risk model. Two hundred and fifty-seven differentially methylated genes were successfully screened out in cancer tissues and normal tissues; 39 related to GO enrichments and 19 related to PATHWAY pathways were found; the best prognostic risk model was obtained, risk score $=Q R F P$ (degree of methylation) $\times(-3.657)+S 100 A 16 \times(-3.378)+$ TDRD1 x (-4.001) + $S M O \times$ ( 3.548$)$; it was determined from each sample that the median value of the risk score was 0.936; using it as the cut-off value, the five-year survival rate in high-risk group of patients was $72.4 \%$ (95\% CI, 62.7-83.6\%), and that in low-risk group of patients was $86.6 \%$ (95\% CI, 78.6-95.3\%). The difference in the survival rate between the high-risk and low-risk groups was significant $(\mathrm{P}<0.001)$. The AUC of ROC curve was 0.791 , so the model had a good clinical application value. This study
\end{abstract}

Correspondence to: Dr Feng Jin, Department of Breast Surgery, The First Affiliated Hospital of China Medical University, 155 Nanjing North Street, Heping, Shenyang, Liaoning 110001, P.R. China

E-mail: ff37ta@163.com; jinfeng@cmu.edu.cn

*Contributed equally

Key words: TCGA, breast cancer, methylation, GO enrichment, PATHWAY, prognostic risk model successfully found multiple breast cancer-related methylation genes, the relationship between them and the course and prognosis of breast cancer was analyzed. Moreover, a prognostic risk model was constructed, which facilitated the expansion of the current study on the role of methylation in the occurrence and development of breast cancer.

\section{Introduction}

Breast cancer is a malignant tumor in the mammary gland tissue (1). Orthotopic breast cancer is not fatal, but its cells are easy to fall off, and these shedding cancer cells will be free from blood or lymph fluid and spread throughout the body to form cancer, then cancer metastasis is formed, thereby threatening life (2). The incidence of breast cancer has been on an upward trend, and in statistics of DeSantis et al (3), 1 out of 10 women in the United States has breast cancer. Although China is not a country with a high incidence of breast cancer, the growth rate in China has been approximately 2 percentage points higher than that in some high-incidence countries in recent years (4). In the recently published data (5), the incidence of breast cancer still ranks first among female malignant tumors in the cancer registration in China. The peak of onset age of breast cancer is approximately 53 years, but now it tends to be younger (6).

Methylation is an important modification of protein and nucleic acid and one of the most important research topics in epigenetics (7). In recent years, methylation has been studied in the diagnosis, efficacy and prognostic evaluation of various cancers such as ovarian cancer (8), cervical cancer (9) and hepatocellular carcinoma (10). The clinical application of methylation in breast cancer has also been systematically studied. The report of An et al (11) proposed that the methylation of MGMT gene that was closely related to the clinical stage, histological grade and lymph node metastasis of breast cancer played an important role in its progression. Studies of Hao et al (12) showed that the combined detection of the methylation degree of a variety of genes could be a good judgement of tumor stage and lymph node metastasis. However, these studies have focused on a small number of candidate genes and have not systematically screened out methylation genes that may be related to the occurrence and development of 
breast cancer. The Cancer Genome Atlas (TCGA) (13) and the Gene Expression Omnibus (GEO) (14) are commonly used public databases in bioinformatics analysis, but the former has complete patient data and is more conducive to the related analysis of the course and prognosis. Therefore, we hope to expand the current study on the role of methylation in the occurrence and development of breast cancer by screening out breast cancer-related methylation genes from the TCGA database, and analyzing the relationship between them and the course and prognosis of breast cancer.

\section{Materials and methods}

Data collection and preprocessing. The data were downloaded from the TCGA database. The RNA-seq data and methylation data for level 3 were downloaded from TCGA, and the selected samples were all patient tissue samples. First, the RNA-seq data files were merged into a matrix file using the merge script of the Perl language (http://www.perl.org/). Then, the gene name was converted from the Ensembl id to the matrix of the gene symbol through the Ensembl database (http://asia.ensembl.org/index.html). At the same time, the methylation data were merged into a single file through the merge script of the Perl language. In the downloaded RNA-seq data and methylation data, the data with incomplete clinical information were excluded. Only the samples that had undergone RNA sequencing and methylated chip data were retained in the remaining data to make it possible to perform a linkage analysis of transcription and methylation.

The study was approved by the Ethics Committee of The First Affiliated Hospital of China Medical University (Shenyang, China).

Screening of differentially methylated genes. All cancer tissues and normal tissues were compared and all high and low methylated genes were looked for $(\mathrm{FDR}<0.05)$ using the MethylMix R package (http://www.bioconductor.org/packages/release/bioc/html/MethylMix.html) in the R Project for Statistical Computing software (Sax software; SAS Institute Inc., Cary, NC, USA). Bidirectional hierarchical clustering of differentially methylated genes was performed, and the differential distribution map of the genes with the most significant methylation difference screened out was plotted using pheatmap R package (https://cran.r-project.org/web/packages/ pheatmap/), and the distribution of methylation degree of cancer samples relative to normal tissues was observed. The correlation between the gene methylation degree and the corresponding gene expression was calculated using Pearson's correlation test in the cor.test function of the $\mathrm{R}$ language (https://www.r-project.org/) (filter condition was cor $<-0.3$ and $\mathrm{P}<0.05)$.

GO enrichment analysis. The GO enrichment analysis of differentially methylated genes was performed using DAVID (Database for Annotation, Visualization and Integration Discovery). First, the DAVID database was logged in (https:// david.ncifcrf.gov/), the Functional Annotation was selected, and the list of differentially expressed genes was submitted. Then, the OFFICIAL_GENE_SYMBOL in the Select Identifier was selected, and the Gene List in List Type was
Table I. Clinical data of samples.

\begin{tabular}{lcc}
\hline Covariates & Type & No. of patients [n (\%)] \\
\hline Survival status & Alive & $616(91.94 \%)$ \\
T & Dead & $54(8.06 \%)$ \\
& T1 & $175(26.12 \%)$ \\
& T2 & $381(56.87 \%)$ \\
N & T3 & $93(13.88 \%)$ \\
& T4 & $21(3.13 \%)$ \\
& N0 & $306(45.67 \%)$ \\
& N1 & $233(34.78 \%)$ \\
M & N2 & $76(11.34 \%)$ \\
& N3 & $49(7.31 \%)$ \\
Stage & NX & $6(0.90 \%)$ \\
& M0 & $530(79.10 \%)$ \\
& M1 & $11(1.64 \%)$ \\
& MX & $129(19.25 \%)$ \\
Age & Stage I & $112(16.72 \%)$ \\
& Stage II & $380(56.72 \%)$ \\
& Stage III & $167(24.93 \%)$ \\
& Stage IV & $11(1.64 \%)$ \\
& $\leq 65$ & $482(71.94 \%)$ \\
& $>65$ & $188(28.06 \%)$ \\
\hline
\end{tabular}

selected, and the Submit List was clicked finally. At the same time, the figure of enrichment results was plotted using the GOplot R package (https://cran.r-project.org/web/packages/ GOplot/).

PATHWAY analysis. The differentially methylated genes were analyzed using the over-representation analysis function of ConsensusPathDB (http://cpdb.molgen.mpg.de/). The PATHWAY pathways enrichment analysis of differentially methylated genes was performed using the KEGG database. $\mathrm{P}<0.05$ was the screening condition.

Single factor and multivariate Cox analysis. To determine the methylation genes related to survival, single factor Cox analysis of differentially methylated genes was performed using Survival R package (https://cran.r-project.org/web/ views/Survival.html), and selection of differentially methylated genes with $\mathrm{P}<0.05$ in the single factor analysis for subsequent multivariate analysis was performed. The optimal risk model was found based on the Akaike Information Criterion (AIC) (15).

Survival curve and ROC curve plotting. According to the optimal risk model obtained from the multivariate Cox analysis and the gene methylation degree of each sample, the survival score was performed, and the median value of risk score of each sample was calculated. The patients above the median value were in the high-risk group, patients below it in the low-risk group. The survival curves of the two groups were plotted using the Kaplan-Meier method, and the difference between them was tested using the log-rank method. The ROC 


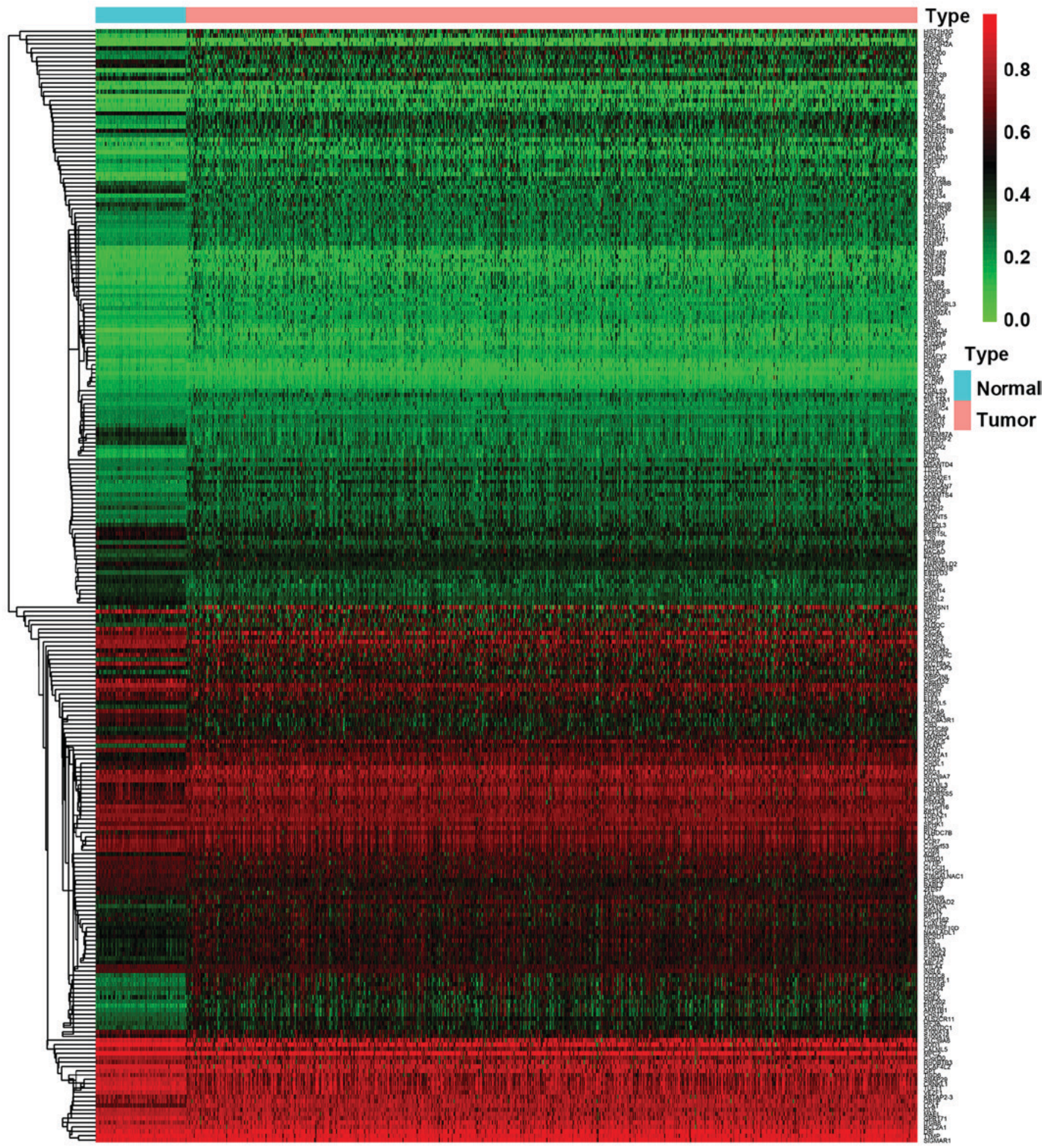

Figure 1. Thermal map analysis of differentially methylated genes. The pheatmap R package was used for bidirectional hierarchical clustering of differentially methylated genes in breast cancer and adjacent tissues. In the figure, red indicates that the gene is highly methylated in the sample, and green low methylated in the sample.

curve was plotted to predict the value of the patient's survival time through the gene methylation degree.

\section{Results}

Clinical data of samples. According to the inclusion criteria we set, 670 samples were finally obtained as the subjects, and the clinical data statistics are shown in Table I.
Screening of differentially methylated genes. Through a comparison of the gene methylation levels in cancer and normal tissues, 257 differentially methylated genes were screened out $($ FDR $<0.05)$ and the thermal map was plotted (Fig. 1), in which there were 161 genes with higher methylation degree of cancer tissues than that of normal tissues, and 96 genes with it lower than that of normal tissues. The FDR (corrected P-value) was used as the standard and the first 10 differentially methylated 

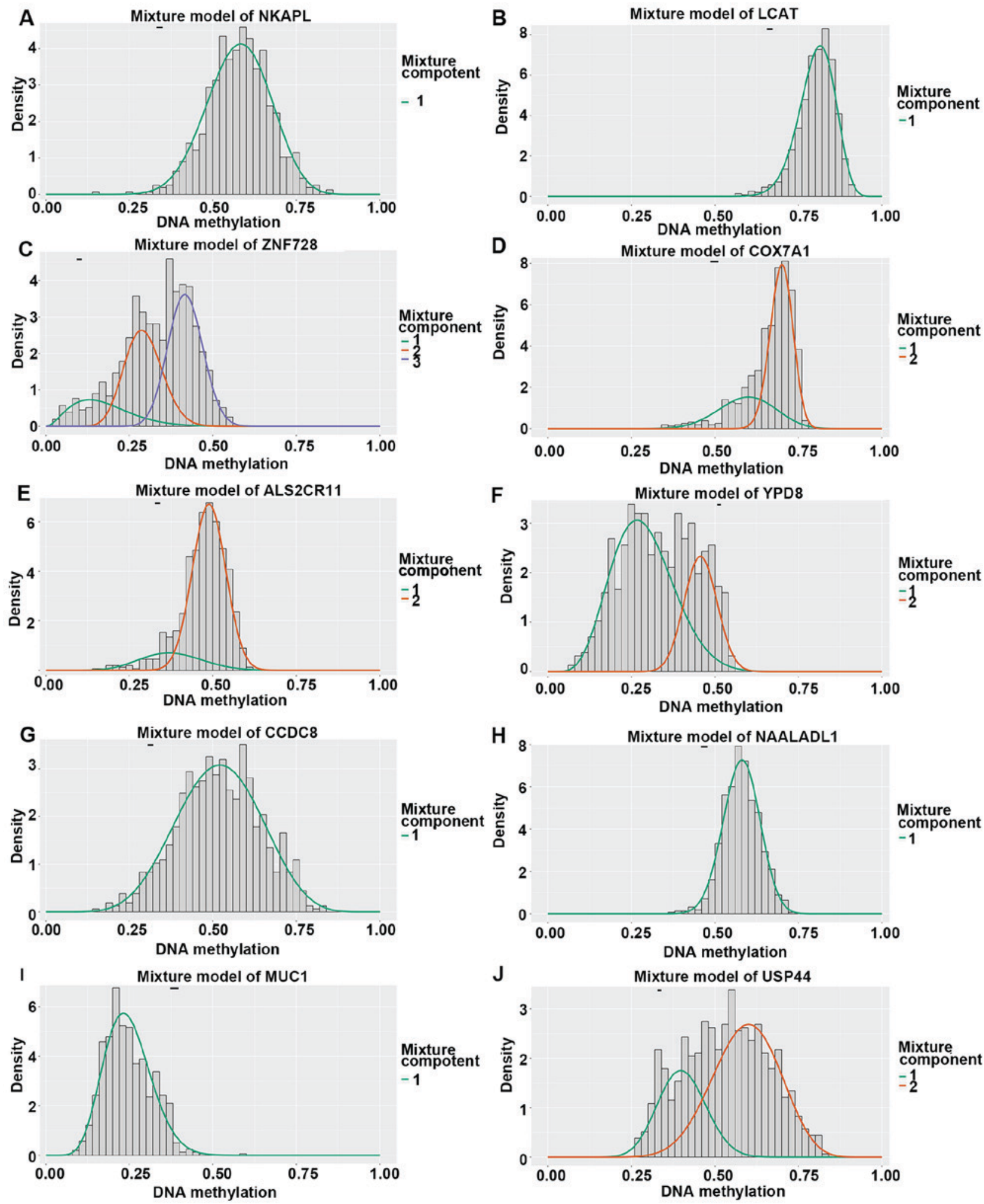

Figure 2. Distribution map of methylation degree of partially differentially methylated genes (A-J). The abscissa is the methylation degree, the ordinate is the number of methylation samples, the histogram represents the methylation distribution of cancer group, and the curve is the simulated trend curve of the methylation distribution of cancer group. The black horizontal line above the figure is the methylation level distribution of the normal sample. The distribution of methylation degree of the cancer sample relative to the normal sample can be clearly seen from the figure.

genes with the smallest P-value were selected (Table II). The distribution map of methylation degree was plotted (Fig. 2A-J).

Correlation analysis between methylation degree and gene expression. Correlation analysis between methylation degree of
257 differentially methylated genes and their gene expression was performed, and it was found that the methylation degree of these 257 genes was negatively correlated with their expression. The higher the methylation degree was, the lower the gene expression. Based on the P-value obtained from the 

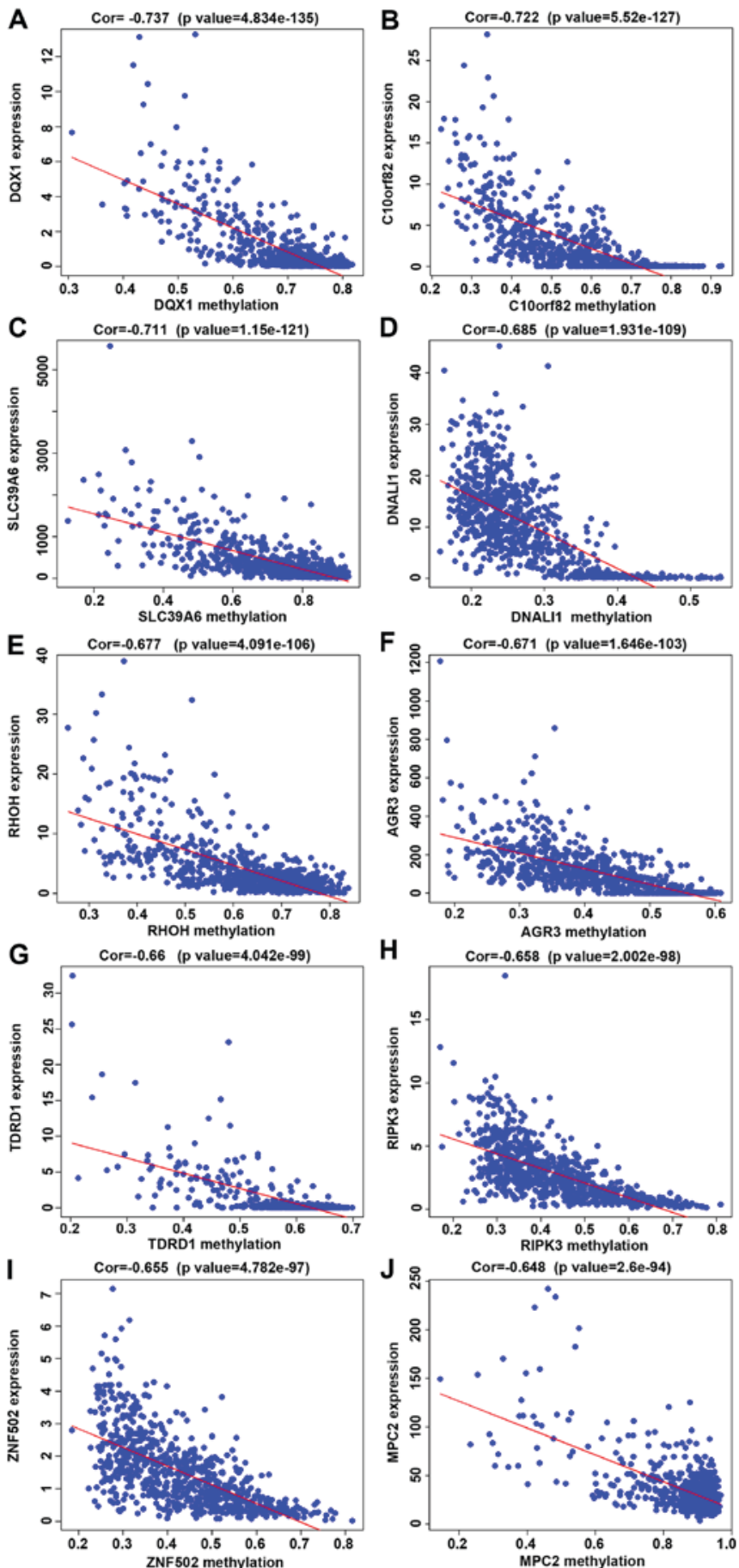

Figure 3. Correlation between methylation degree of partially differentially methylated genes and gene expression (A-J). Pearson's correlation analysis was used for analysis of the correlation between the methylation degree and gene expression. It can be seen from the figure that the gene methylation degree is negatively correlated with the expression. The abscissa is the beta value of the gene methylation degree, and the ordinate is the expression of the gene. Cor is the correlation coefficient and P-value is the test value of correlation.

Pearson's correlation test, the first 10 genes with the smallest P-value were selected (Table III), and the correlation figure was plotted (Fig. 3A-J).

GO enrichment analysis. The GO enrichment analysis of 257 differentially methylated genes was performed using
DAVID, and results showed that the most relevant enrichments were 'extracellular exosome', 'superoxide dismutase activity', 'intracellular', 'mast cell granule' and 'glutathione derivative biosynthetic', (Table IV and Fig. 4).

PATHWAY analysis. The PATHWAY pathways enrichment analysis of 257 differentially methylated genes was performed using ConsensusPathDB, and a total of 19 related PATHWAYs were found $(\mathrm{P}<0.05)$, among which the most relevant were 'D-Glutamine and D-glutamate metabolism', 'Estrogen signaling pathway' and 'Fluid shear stress and atherosclerosis' (Table V and Fig. 5).

Single factor and multivariate Cox analysis. Single factor Cox analysis of differentially methylated genes was performed using the Survival $\mathrm{R}$ package, the screening condition was $\mathrm{P}<0.01$, and 14 genes were obtained (Table VI). At the risk ratio $(\mathrm{HR})>1$, the higher the gene expression was, the higher the risk was; at $\mathrm{HR}<1$, the higher the gene expression was, the lower the risk was. Multivariate analysis of 14 selected genes significantly different from single factor was performed using Survival package. The optimal model was found according to AIC and four optimal gene models were obtained. The risk model obtained was: risk score $=Q R F P$ (Degree of methylation) $\times(-3.657)+S 100 A 16 \times(-3.378)+T D R D 1 \times(-4.001)+$ $S M O \times(3.548)$.

Survival curve and ROC curve plotting. According to the optimal risk model obtained from the multivariate Cox analysis and the degree of gene methylation of each sample, the survival score was performed. The median value of risk score of each sample was calculated to be 0.936 , and used as the cut-off value, 335 patients with a risk score $>0.936$ were in the high-risk group and 335 patients $<0.936$ in the low-risk group. Based on the high-risk and low-risk groups, the survival curve was plotted using the Kaplan-Meier method (Fig. 6). From the survival data, we could see that the five-year survival rate in the high-risk group of patients was 72.4\% (95\% CI, 62.7-83.6\%), and that in the low-risk group of patients was $86.6 \%(95 \%$ CI, 78.6-95.3\%), and the difference thereof between the two groups was significant $(\mathrm{P}<0.001)$. At the same time, the ROC curve was plotted (Fig. 7) and AUC was 0.791, indicating that our model could well predict patient survival.

\section{Discussion}

In this study, 670 samples which had undergone RNA sequencing and methylated chip data were selected by TCGA, in which a differential methylation analysis was performed, and correlation analysis, GO enrichment analysis, PATHWAY analysis, single factor analysis, multivariate analysis, prognostic model and ROC curve were performed on the differentially methylated genes.

Methylation is one of the most important studies in epigenetics. In mammals, DNA methylation mainly occurs on $\mathrm{CpG}$ islands, often in the promoter region or the first exon and the $3^{\prime}$ end of the gene (16), and about $70 \%$ of human gene promoters exist in $\mathrm{CpG}$ islands (17). Studies have found that almost all tumors can find abnormal DNA methylation in comparison between cancer tissues and corresponding 
Table II. Partially differentially methylated genes.

\begin{tabular}{|c|c|c|c|c|c|}
\hline Gene symbol & $\begin{array}{l}\text { Normal group } \\
\text { methylation level (A) }\end{array}$ & $\begin{array}{c}\text { Cancer group } \\
\text { methylation level (B) }\end{array}$ & $\begin{array}{l}\text { Methylation } \\
\text { degree }\end{array}$ & P-value & FDR \\
\hline$N K A P L$ & 0.340837653 & 0.577383257 & 0.236545604 & $4.31 \mathrm{E}-54$ & $1.22 \mathrm{E}-51$ \\
\hline$L C A T$ & 0.664095202 & 0.803431309 & 0.139336107 & $1.16 \mathrm{E}-50$ & $1.64 \mathrm{E}-48$ \\
\hline ZNF728 & 0.099901799 & 0.333781489 & 0.233879691 & $1.24 \mathrm{E}-49$ & $1.16 \mathrm{E}-47$ \\
\hline$C O X 7 A 1$ & 0.498567597 & 0.664727174 & 0.166159577 & $2.04 \mathrm{E}-46$ & $1.44 \mathrm{E}-44$ \\
\hline$A L S 2 C R 11$ & 0.334427241 & 0.470741226 & 0.136313985 & 4.83E-46 & $2.72 \mathrm{E}-44$ \\
\hline LYPD8 & 0.512159389 & 0.335695176 & -0.176464213 & $9.37 \mathrm{E}-46$ & $4.40 \mathrm{E}-44$ \\
\hline$C C D C 8$ & 0.312900607 & 0.518293085 & 0.205392479 & $7.78 \mathrm{E}-45$ & $3.09 \mathrm{E}-43$ \\
\hline NAALADL1 & 0.467929203 & 0.578828343 & 0.110899139 & $8.77 \mathrm{E}-45$ & $3.09 \mathrm{E}-43$ \\
\hline$M U C 1$ & 0.384572295 & 0.246245302 & -0.138326993 & $1.13 \mathrm{E}-43$ & $3.54 \mathrm{E}-42$ \\
\hline USP44 & 0.333728357 & 0.532191383 & 0.198463026 & $1.97 \mathrm{E}-43$ & $5.56 \mathrm{E}-42$ \\
\hline
\end{tabular}

Methylation degree, B-A.

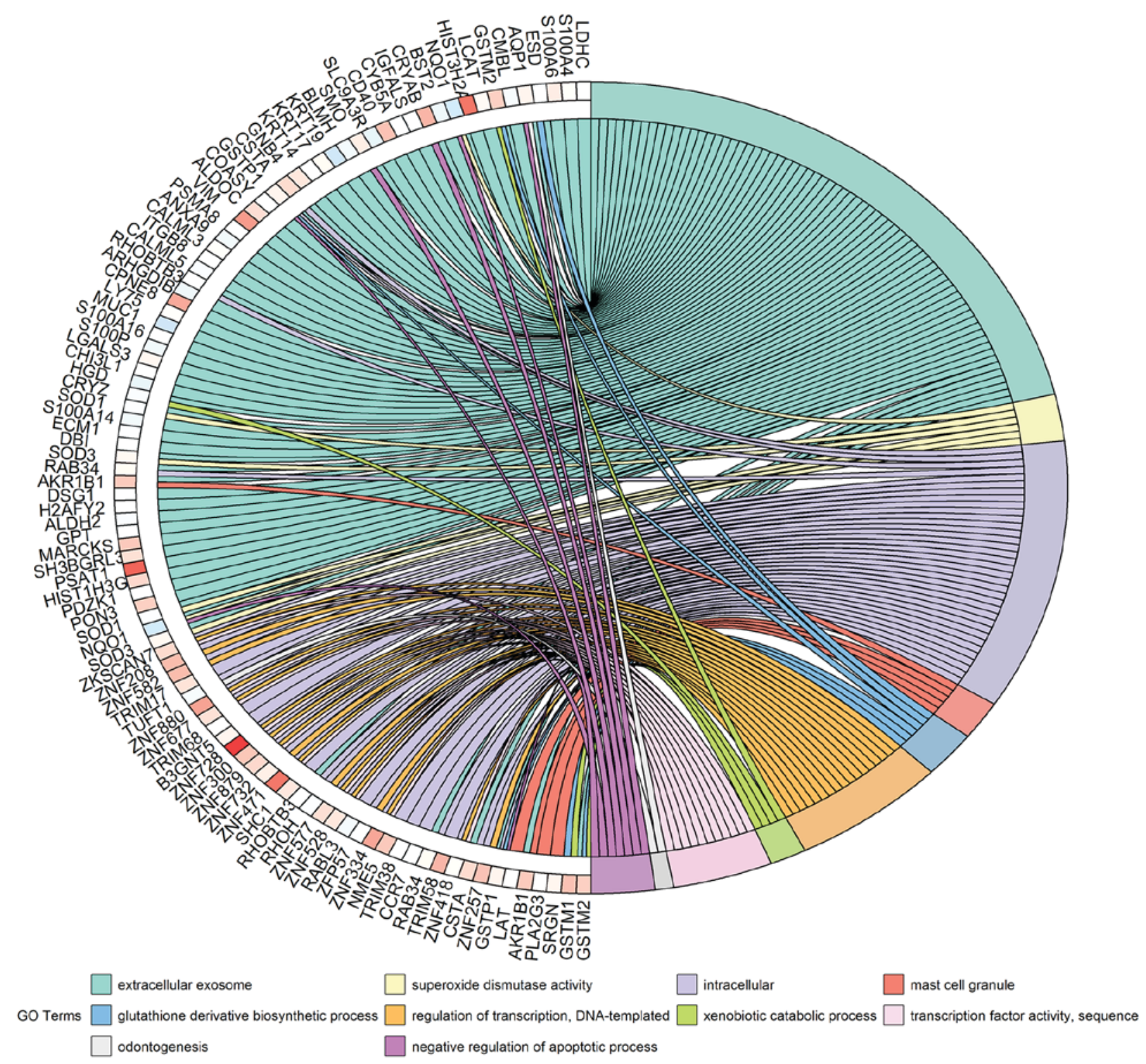

Figure 4. GO enrichment analysis of differentially methylated genes. The left side of the circle is the gene and the right side is the pathway. Different colors represent different pathways, and the color of each pathway is annotated below the circle. If the gene belongs to a pathway, there will be a line between the gene and the pathway. 


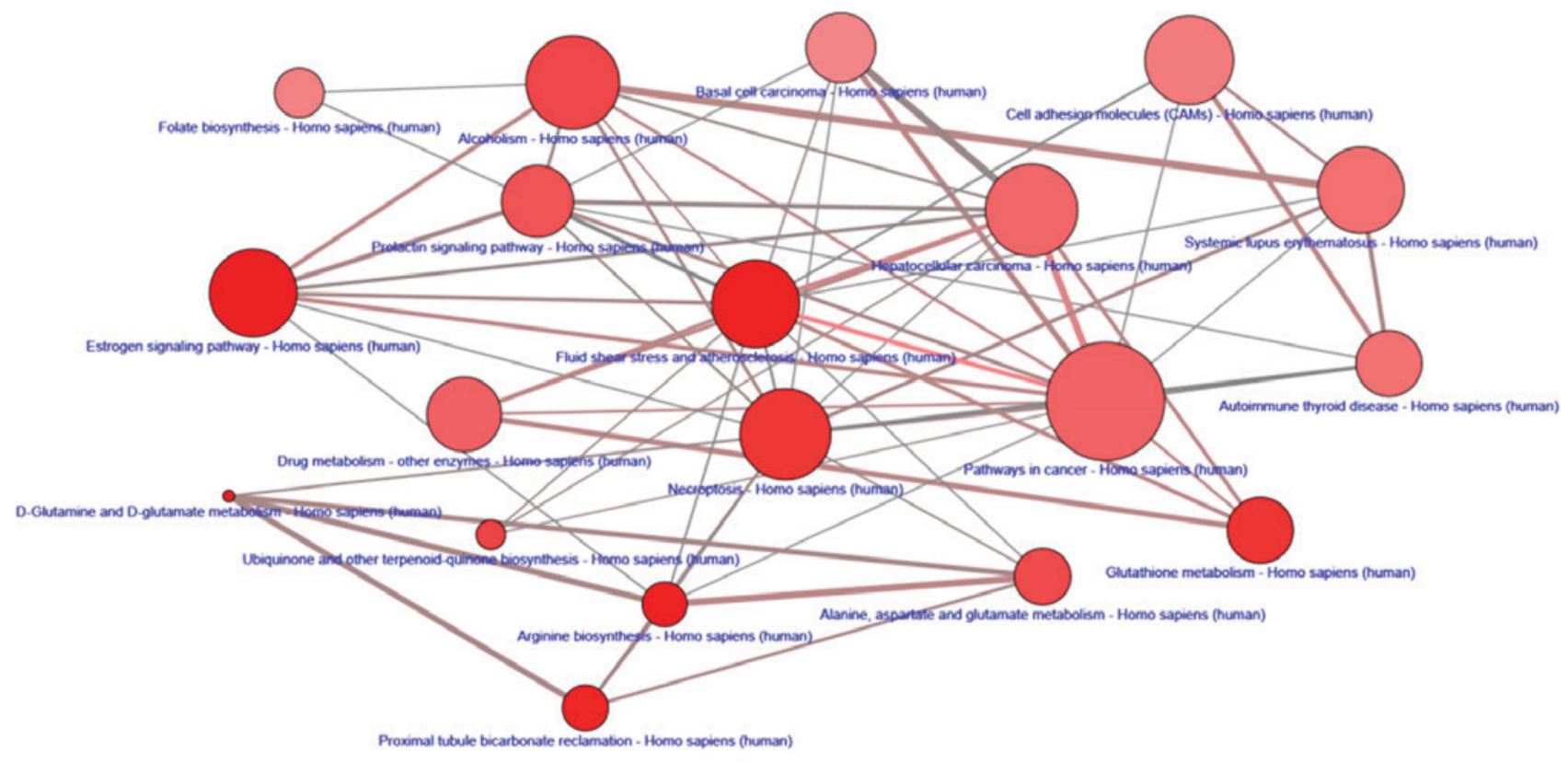

\begin{abstract}
Node label color
The node label color denotes the type of the gene sets:

neighborhood-based set manually curated pathway Gene Ontology category protein complex
\end{abstract}

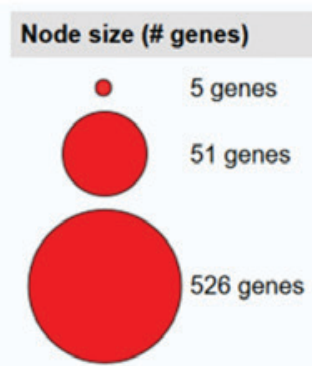

Edge width ( $\%$ shared genes)

Node color ( $p$ value)

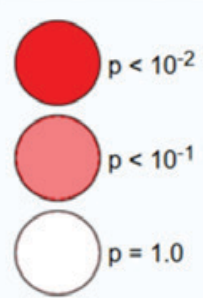

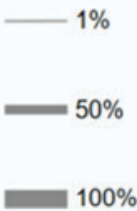

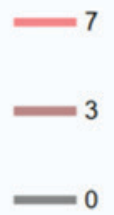

Figure 5. PATHWAY analysis of differentially methylated genes. The circle in the figure represents the pathway, and the line represents the relationship between pathways. The size of the circle represents the number of genes in the pathway. The larger the number is, the more the number of pathway gene data is; the color of the circle represents an enriched significant P-value, and the stronger red represents a higher degree of enrichment. The bold line represents the number of genes with the same pathway and the line color represents the number of genes with the same difference before the pathway.

Table III. Correlation analysis between methylation degree and gene expression.

\begin{tabular}{lll}
\hline Gene & \multicolumn{1}{c}{ cor } & P-value \\
\hline DQX1 & -0.737068974 & $4.83 \mathrm{E}-135$ \\
C10orf82 & -0.721853363 & $5.52 \mathrm{E}-127$ \\
SLC39A6 & -0.71121904 & $1.15 \mathrm{E}-121$ \\
DNALI1 & -0.684796632 & $1.93 \mathrm{E}-109$ \\
RHOH & -0.677085189 & $4.09 \mathrm{E}-106$ \\
AGR3 & -0.670876546 & $1.65 \mathrm{E}-103$ \\
TDRD1 & -0.660058794 & $4.04 \mathrm{E}-99$ \\
RIPK3 & -0.658304073 & $2.00 \mathrm{E}-98$ \\
ZNF502 & -0.654789106 & $4.78 \mathrm{E}-97$ \\
MPC2 & -0.64767024 & $2.60 \mathrm{E}-94$ \\
\hline
\end{tabular}

non-tumor normal tissues (18). Therefore, methylation is a very important part in the current study on the molecular level of cancer.

In our study, a total of 257 differentially methylated genes were found by comparison between breast cancer tissues and

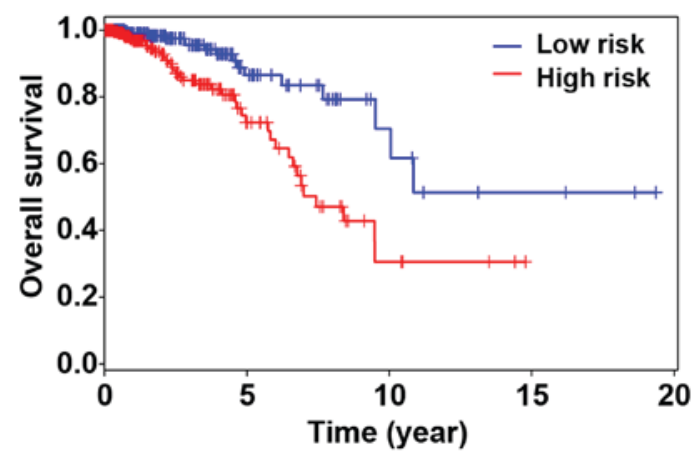

Figure 6. Survival curve. The patients were divided into high-risk group $(>0.936)$ and low-risk group $(<0.936)$ according to the median value:-0.936 of risk score. The Kaplan-Meier method was used to plot the survival curves of the two groups, and the log-rank method was used to test the difference between them that was significant in the survival rate between the high-risk and low-risk groups $(\mathrm{P}<0.001)$.

their corresponding normal tissues, of which the methylation degree of $N K A P L$ was the highest. In a study on 5 liver cancer cell lines and 62 pairs of primary liver cancer and its adjacent non-cancerous liver tissues, $\mathrm{Ng}$ et al (19) found that NKAPL was highly methylated in liver cancer, and 
Table IV. GO enrichment analysis of differentially methylated genes.

\begin{tabular}{lcrrr}
\hline Term & Enrichments & Count & P-value & FDR \\
\hline GO:0070062 & Extracellular exosome & 60 & $8.25 \mathrm{E}-05$ & 0.10545219 \\
GO:0004784 & Superoxide dismutase activity & 3 & 0.001692298 & 2.353503638 \\
GO:0005622 & Intracellular & 31 & 0.002142855 & 2.7070955 \\
GO:0042629 & Mast cell granule & 4 & 0.002400191 & 3.027607057 \\
GO:1901687 & Glutathione derivative biosynthetic process & 4 & 0.002628416 & 4.227224422 \\
GO:0006355 & Regulation of transcription, DNA-templated & 33 & 0.002759092 & 4.43294563 \\
GO:0042178 & Xenobiotic catabolic process & 3 & 0.003254731 & 5.209453579 \\
GO:0003700 & Transcription factor activity, sequence-specific DNA binding & 24 & 0.004284423 & 5.858842135 \\
GO:0042476 & Odontogenesis & 4 & 0.004764259 & 7.538061353 \\
GO:0043066 & Negative regulation of apoptotic process & 14 & 0.005694098 & 8.94558752 \\
\hline
\end{tabular}

Table V. Partial PATHWAY enrichment analysis.

\begin{tabular}{|c|c|c|c|}
\hline Pathway ID & Pathway & P-value & Enriched genes \\
\hline hsa00471 & D-Glutamine and D-glutamate metabolism & 0.001650165 & $G L S ; G L U D 1$ \\
\hline hsa04915 & Estrogen signaling pathway & 0.002030577 & $\begin{array}{l}\text { SHC1; ESR1; CALML3; CALML5; KRT17; } \\
\text { KRT14; KRT19 }\end{array}$ \\
\hline hsa05418 & Fluid shear stress and atherosclerosis & 0.00229801 & $\begin{array}{l}\text { BMP4; CALML3; GSTM1; GSTM2; } \\
\text { CALML5; NQO1; GSTP1 }\end{array}$ \\
\hline hsa00220 & Arginine biosynthesis & 0.002443407 & $G P T ; G L S ; G L U D 1$ \\
\hline hsa04964 & Proximal tubule bicarbonate reclamation & 0.003192003 & $G L S ; G L U D 1 ; A Q P 1$ \\
\hline hsa00480 & Glutathione metabolism & 0.005331216 & GSTM1; GSTM2; GSTP1; GPX7 \\
\hline hsa04217 & Necroptosis & 0.005718681 & $\begin{array}{l}\text { H2AFY2; RIPK3; GLUD1; STAT5A; IFNGR2; } \\
\text { TNFRSF10D; HIST3H2A }\end{array}$ \\
\hline hsa00130 & $\begin{array}{l}\text { Ubiquinone and other terpenoid-quinone } \\
\text { biosynthesis }\end{array}$ & 0.008620151 & $T A T ; N Q O 1$ \\
\hline hsa05034 & Alcoholism & 0.009346881 & $\begin{array}{l}\text { SHC1; CALML3; GNB4; H2AFY2; CALML5; } \\
\text { HIST1H3G; HIST3H2A }\end{array}$ \\
\hline hsa00250 & Alanine, aspartate and glutamate metabolism & 0.010523928 & GPT; GLS; GLUD1 \\
\hline
\end{tabular}

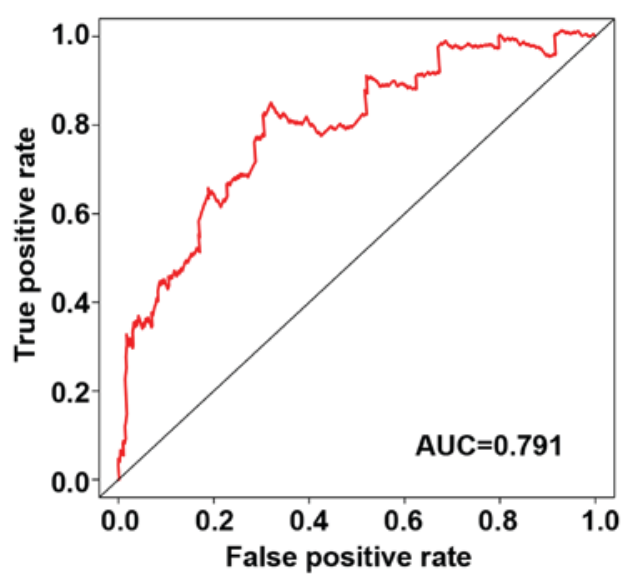

Figure 7. ROC curve figure. In the ROC curve analysis, it was obtained that AUC was 0.791, indicating that our model can well predict patient survival. The larger the AUC value is, the more likely the current classification algorithm is to place the positive sample in front of the negative sample, i.e. for better classification. the methylation degree was negatively correlated with its expression level. It could also inhibit the growth of cancer cells in liver cancer cells, which was a potential prognostic marker. In our study, $N K A P L$ was also highly methylated in breast cancer tissues, and the methylation degree was also negatively correlated with its expression level. It suggested that NKAPL may be involved in mechanism of cancer suppression in breast cancer tissues. At the same time, NKAPL was also enriched in 'regulation of transcription, DNA-templated' and we hypothesized that it may affect cancer cell changes by affecting the transcription and regulation of DNA. However, there have been no reports of $N K A P L$ in breast cancer-related studies. In the follow-up GO enrichment analysis, it was found that 'extracellular exosome' and 'superoxide dismutase activity' were the most relevant enrichments with differentially methylated genes, which mainly affected the activity of extracellular body and superoxide dismutase (SOD). 
Table VI. Single factor Cox analysis.

\begin{tabular}{lccc}
\hline Gene & HR & $\mathrm{z}$ & P-value \\
\hline QRFP & 0.047697004 & -3.632634369 & 0.000280542 \\
CSTA & 0.062945881 & -3.360829859 & 0.000777087 \\
ELF5 & 0.058117795 & -3.264669409 & 0.001095919 \\
GRHL2 & $4.13 \mathrm{E}-06$ & -3.167428425 & 0.001537936 \\
CCDC89 & 0.024575046 & -2.971482358 & 0.002963659 \\
S100A3 & 0.011686928 & -2.86232633 & 0.004205437 \\
S100A16 & 0.024716952 & -2.82925571 & 0.00466564 \\
TDRD1 & 0.017847438 & -2.791779478 & 0.005241907 \\
SMO & 42.93347544 & 2.704736969 & 0.006835849 \\
CALML5 & 0.054952261 & -2.672962518 & 0.007518465 \\
SCG5 & 0.015402449 & -2.669608387 & 0.007593976 \\
TAF1D & 0.040537383 & -2.661547052 & 0.007778247 \\
SOD1 & 0.145404134 & -2.6286443 & 0.008572598 \\
KRTAP2-3 & 0.04690311 & -2.604144621 & 0.009210388 \\
\hline
\end{tabular}

Thirty years ago, when the first contact with the extracellular body was made, it was originally thought that the cell component was discarded or unwanted. Recently, it was realized that the extracellular body contains cell-specific proteins, lipids and genetic material that can be passed to distant tissues and cells, thereby changing their function and physiology (20). Urabe et al (21) believed that the extracellular body was expected to become a liquid biomarker for prostate cancer, kidney cancer and bladder cancer by regulating the immune system and angiogenesis that affect cancerous changes. At the same time, Chen et al (22) thought that the extracellular body induced the occurrence and recurrence of liver cancer cells through the MAPK/ERK signaling pathway. In our study, methylation genes influenced changes in the extracellular body and thus affected changes in breast cancer, but specific experiments are needed to confirm this conjecture. SOD can scavenge superoxide anion radicals, thereby inhibiting the occurrence of lipid peroxidation, autoimmune diseases and tumors (23).

In study of Kocot et al (24), it was believed that SOD was closely related to the metastasis and differentiation of colorectal cancer, and had certain application value in its treatment. According to the characteristics of SOD, we believed that its activity was related to the occurrence of cancer, and it was not comprehensive in the study on breast cancer. The results of this study again suggest the importance of SOD, and it is necessary to further discuss its influencing mechanism. In the PATHWAY analysis, the most closely related to the differentially methylated genes in breast cancer tissues was the 'D-Glutamine and D-glutamate metabolism'. Glutamine is an important fuel for the immune system and has important immunomodulatory effects (25), which can promote the division and differentiation of lymphocytes and macrophages. Exogenous glutamine can significantly increase the number of lymphocytes, $\mathrm{T}$ lymphocytes and CD4/CD8 ratio in critically ill patients and enhance the body immunity (26). Many cancers are very dependent on glutamine (27), transcriptional programs of which drives its high consumption, so it is called 'glutamine metabolism addiction' (28). However, whether this phenomenon exists in breast cancer still needs further study.
In the follow-up risk model construction and ROC curve judgement, the best risk model was obtained, risk score $=$ QRFP $\times(-3.657)+$ S100A16 $\times(-3.378)+$ TDRD1 $\times(-4.001)+$ $S M O \times$ (3.548); with the median value- 0.936 of risk score as the cut-off value, the 5-year survival rate in high-risk group of patients (risk score $>0.936$ ) was $72.4 \%$ (95\% CI, 62.7-83.6\%), and that in low-risk group of patients was $86.6 \%$ (95\% CI, 78.6-95.3\%) and AUC was 0.791 as judged by ROC curve, having a good application value. By obtaining the methylation degree of the patient's QRFP, S100A16, TDRDI and SMO in the clinic and it can be calculated whether the patient is in a high-risk or low-risk state and can predict its five-year survival rate, as a reminder for targeted treatment.

The main drawback of this study is that it only uses computer simulation data but fails to verify the results by specific clinical data and to perform specific cancer cell and animal model experiments. In addition, there are major differences in the regions and human races. The advantage behind these drawbacks lies in the fact that molecular bioinformatics has made it more efficient to spend time, resources and manpower on the molecular area. In future studies, we will conduct a more in-depth study on the differentially methylated genes screened out and the related GO and PATHWAY.

After long-term calculations and discussions, we finally concluded that the occurrence and development of breast cancer were closely correlated with methylation genes such as NKAPL, QRFP, S100A16, TDRDI and SMO and related biological processes and signaling pathways such as 'extracellular exosome', 'superoxide dismutase activity' and 'D-Glutamine and D-glutamate metabolism'. We will conduct more in-depth studies on these aspects as conditions permit. Recently, there have been few studies on the breast cancerrelated gene methylation; thus, we hope that our experimental results can enrich the study in this area and provide help for clinical diagnosis and treatment in the future.

\section{Acknowledgements}

Not applicable.

\section{Funding}

No funding was received.

\section{Availability of data and materials}

The datasets used and/or analyzed during the present study are available from the corresponding author on reasonable request.

\section{Authors' contributions}

LF and FJ drafted the manuscript. LF helped with GO enrichment analysis. FJ contributed to PATHWAY analysis. Both authors have read and approved the final manuscript.

\section{Ethics approval and consent to participate}

The study was approved by the Ethics Committee of The First Affiliated Hospital of China Medical University (Shenyang, China). 


\section{Patient consent for publication}

Not applicable.

\section{Competing interests}

The authors declare that they have no competing interests.

\section{References}

1. Inoue M, Nakagomi H, Nakada H, Furuya K, Ikegame K, Watanabe H, Omata M and Oyama T: Specific sites of metastases in invasive lobular carcinoma: A retrospective cohort study of metastatic breast cancer. Breast Cancer 24: 667-672, 2017.

2. Yates LR, Knappskog S, Wedge D, Farmery JHR, Gonzalez S, Martincorena I, Alexandrov LB, Van Loo P, Haugland HK, Lilleng PK, et al: Genomic evolution of breast cancer metastasis and relapse. Cancer Cell 32: 169-184.e7, 2017.

3. DeSantis CE, Ma J, Goding Sauer A, Newman LA and Jemal A: Breast cancer statistics, 2017, racial disparity in mortality by state. CA Cancer J Clin 67: 439-448, 2017.

4. Xing P, Dong H, Liu Q, Yao F, Xu Y, Chen B, Zheng X, Wu Y, Jin $\mathrm{F}$ and $\mathrm{Li}$ J: Impact of persistence on survival of patients with breast cancer treated with endocrine therapy in Northeast China: A prospective study. Oncotarget 8: 102499-102510, 2017.

5. Zuo TT, Zheng RS, Zeng HM, Zhang SW and Chen WQ: Female breast cancer incidence and mortality in China, 2013. Thorac Cancer 8: 214-218, 2017.

6. McGrath KG: An earlier age of breast cancer diagnosis related to more frequent use of antiperspirants/deodorants and underarm shaving. Eur J Cancer Prev 12: 479-485, 2003.

7. Widschwendter M, Siegmund KD, Müller HM, Fiegl H, Marth C, Müller-Holzner E, Jones PA and Laird PW: Association of breast cancer DNA methylation profiles with hormone receptor status and response to tamoxifen. Cancer Res 64: 3807-3813, 2004.

8. Flanagan JM, Wilson A, Koo C, Masrour N, Gallon J, Loomis E, Flower K, Wilhelm-Benartzi C, Hergovich A, Cunnea P, et al: Platinum-based chemotherapy induces methylation changes in blood DNA associated with overall survival in patients with ovarian cancer. Clin Cancer Res 23: 2213-2222, 2017.

9. Shu R, He J, Wu C and Gao J: The association between RAR $\beta$ and FHIT promoter methylation and the carcinogenesis of patients with cervical carcinoma: A meta-analysis. Tumour Biol 39: 1010428317709126, 2017.

10. Udali S, Guarini P, Ruzzenente A, Ferrarini A, Guglielmi A, Lotto V, Tononi P, Pattini P, Moruzzi S, Campagnaro T, et al: DNA methylation and gene expression profiles show novel regulatory pathways in hepatocellular carcinoma. Clin Epigenetics 7: 43, 2015.

11. An N, Shi Y, Ye P, Pan Z and Long X: Association between MGMT promoter methylation and breast cancer: A metaanalysis. Cell Physiol Biochem 42: 2430-2440, 2017

12. Hao X, Luo H, Krawczyk M, Wei W, Wang W, Wang J, Flagg K, Hou J, Zhang H, Yi S, et al: DNA methylation markers for diagnosis and prognosis of common cancers. Proc Natl Acad Sci USA 114: 7414-7419, 2017.

13. Weinstein JN, Collisson EA, Mills GB, Shaw KR, Ozenberger BA Ellrott K, Shmulevich I, Sander C and Stuart JM; Cancer Genome Atlas Research Network: The Cancer Genome Atlas Pan-Cancer analysis project. Nat Genet 45: 1113-1120, 2013.

14. Xie ZC, Dang YW, Wei DM, Chen P, Tang RX, Huang Q, Liu JH and Luo DZ: Clinical significance and prospective molecular mechanism of MALAT1 in pancreatic cancer exploration: A comprehensive study based on the GeneChip, GEO, Oncomine, and TCGA databases. OncoTargets Ther 10: 3991-4005, 2017.
15. Aho K, Derryberry D and Peterson T: Model selection for ecologists: The worldviews of AIC and BIC. Ecology 95: 631-636, 2014.

16. Stirzaker C, Song JZ, Ng W, Du Q, Armstrong NJ, Locke WJ, Statham AL, French H, Pidsley R, Valdes-Mora F, et al: MethylCpG-binding protein MBD2 plays a key role in maintenance and spread of DNA methylation at CpG islands and shores in cancer. Oncogene 36: 1328-1338, 2017.

17. Kakizaki F, Sonoshita M, Miyoshi H, Itatani Y, Ito S, Kawada K, Sakai Y and Taketo MM: Expression of metastasis suppressor gene AES driven by a Yin Yang (YY) element in a CpG island promoter and transcription factor YY2. Cancer Sci 107: 1622-1631, 2016

18. Das S, Moran B and Perry AS: Assessing DNA methylation in cancer stem cells. Methods Mol Biol 1692: 157-178, 2018.

19. Ng PKS, Lau CPY, Lam EKY, Li SSK, Lui VWY, Yeo W, Ng YK, Lai PBS and Tsui SKW: Hypermethylation of NF- $\kappa B$-activating protein-like $(N K A P L)$ promoter in hepatocellular carcinoma suppresses its expression and predicts a poor prognosis. Dig Dis Sci 63: 676-686, 2018.

20. Roy S, Hochberg FH and Jones PS: Extracellular vesicles: The growth as diagnostics and therapeutics; a survey. J Extracell Vesicles 7: 1438720, 2018

21. Urabe F, Kosaka N, Kimura T, Egawa S and Ochiya T: Extracellular vesicles: Toward a clinical application in urological cancer treatment. Int J Urol 25: 533-543, 2018.

22. Chen L, Guo P, He Y, Chen Z, Chen L, Luo Y, Qi L, Liu Y, Wu Q, Cui Y, et al: HCC-derived exosomes elicit HCC progression and recurrence by epithelial-mesenchymal transition through MAPK/ERK signalling pathway. Cell Death Dis 9: 513, 2018.

23. Landeen KC, Spanos WC and Gromer L: Topical superoxide dismutase in posttreatment fibrosis in patients with head and neck cancer. Head Neck: May 13, 2018. (Epub ahead of print). doi: 10.1002/hed.25119.

24. Kocot J, Kiełczykowska M, Dąbrowski W, Piłat J, Rudzki S and Musik I: Total antioxidant status value and superoxide dismutase activity in human colorectal cancer tissue depending on the stage of the disease: A pilot study. Adv Clin Exp Med 22: 431-437, 2013.

25. Caris AV, Da Silva ET, Dos Santos SA, Tufik S and Dos Santos RVT: Effects of carbohydrate and glutamine supplementation on oral mucosa immunity after strenuous exercise at high altitude: A double-blind randomized trial. Nutrients 9: 692, 2017.

26. Pavlova NN, Hui S, Ghergurovich JM, Fan J, Intlekofer AM, White RM, Rabinowitz JD, Thompson CB and Zhang J: As extracellular glutamine levels decline, asparagine becomes an essential amino acid. Cell Metab 27: 428-438.e5, 2018.

27. Abu Aboud O, Habib SL, Trott J, Stewart B, Liang S, Chaudhari AJ, Sutcliffe J and Weiss RH: Glutamine addiction in kidney cancer suppresses oxidative stress and can be exploited for real-time imaging. Cancer Res 77: 6746-6758, 2017.

28. Chiu M, Taurino G, Bianchi MG, Ottaviani L, Andreoli R, Ciociola T, Lagrasta CAM, Tardito S and Bussolati O: Oligodendroglioma cells lack glutamine synthetase and are auxotrophic for glutamine, but do not depend on glutamine anaplerosis for growth. Int J Mol Sci 19: 19, 2018.

This work is licensed under a Creative Commons Attribution-NonCommercial-NoDerivatives 4.0 International (CC BY-NC-ND 4.0) License. 